\title{
Suitability of OPC UA for distributed energy monitoring
}

\author{
Michael C. KRUTWIG \\ Bucharest University of Economic Studies (ASE), Bucharest, Romania \\ michael@krutwig.com
}

PICBE | 399

\begin{abstract}
According to ISO 50001, energy management systems have become widespread in the manufacturing industry. Especially for most large, energy-intensive companies, an energy management system has become an indispensable component of cost planning and sustainability strategy. Energy monitoring is used for continuously evaluating energy flows. Networked and distributed monitoring in industrial production environments is becoming increasingly common with cyber-physical systems (CPS) and can take advantage of them. Considering this, the OPC Unified Architecture (OPC UA) framework plays a decisive role, as it networks all factory components and creates a standard for interoperability between the components. In a literature review context, this paper examines the flexible OPC UA standard, particularly concerning its applicability to energy monitoring. The research question concerns which OPC UA aspects are of relevance for an energy management system. To reduce the technical resources, a subset of the complete standard tailored to the specific task can also be available in the components. Here, we investigate the requirements an energy-related CPS has to fulfill and extract the standard's relevant components and characteristics from the OPC UA framework based on these requirements. Using the information model of OPC UA, an energy sensor becomes a CPS. We demonstrate this process in a prototypical implementation.
\end{abstract}

Keywords: OPC Unified Architecture, IEC-62541, cyber-physical systems, energy monitoring, sensors.

\section{Introduction}

For companies and organizations, measuring energy consumption is important for energy management. The ISO 50001 standard (Standardization, 2018) describes a management cycle with continuous improvement in energy efficiency. This improvement requires a growing energy flow transparency, which is gathered using more and more measurements. The observation of these energy flows is also called energy monitoring. Growing transparency of energy use is achieved in energy monitoring as an extensive amount of energy sources are measured at progressively more points in short intervals. Further, data from third-party systems must be involved to form suitable energy performance indicators (EnPIs). Networked and automatically read sensors are also required. The theoretical optimum-continuous measurement of all media for all consumers, producers, and energy converters - is hardly achievable for technical and economic reasons. Therefore, the challenge in energy management lies in anticipating where economic efficiency measures will be possible and verifying this potential by supplementary submetering (Rao, Muller, \& Gunn, 2017).

The Internet of Things (IoT) is helpful with this challenge, as there exist sensors that follow the concept of cyber-physical systems (CPSs) (Baheti \& Gill, 2011; Monostori et al., 2016; Schlick, 2012; Wolf, 2009). Such sensors are networked, can be implemented costeffectively, and even fulfill additional functions in an intelligently networked production environment. A CPS, as well as being able to communicate via networking, provides a virtual representation of itself. This virtual twin provides relevant information about states and offers services by calling provided methods. Networking between CPS enables additional, 
higher-level CPS, which allows for creating flexible production processes and innovative services (ElMaraghy, 2005) that can bring companies' additional value (Jeschke, Brecher, Song, \& Rawat, 2017). In the energy sector, CPSs are used to form intelligent power grids (smart grids). These are referred to as cyber-physical energy systems (CPES).

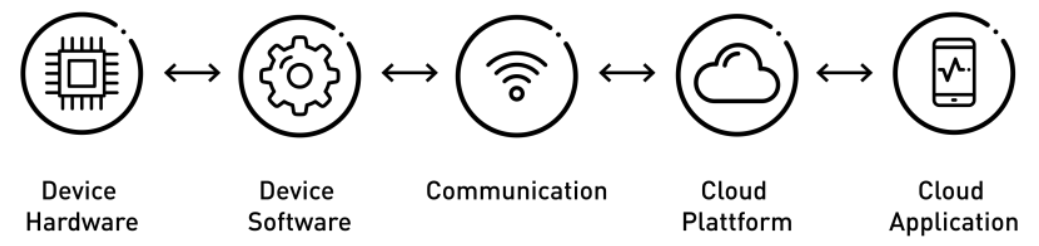

PICBE $\mid 400$

Figure 1. The IoT technology stack

Source: author.

CPS sensors are networked according to the general IoT technology stack (Figure 1), in which sensor data is transferred via a cloud platform to the cloud application. In our case, this is an energy management system. Bi-directional communication between the layers shown in the stack also illustrates the difference between energy monitoring and energy management. In monitoring, one direction to the application would be sufficient. However, in energy management, it should also be possible to control aggregates via switching actuators, for example, to reduce peak loads.

Standards can be used to convert a sensor into a CPS. OPC Unified Architecture (OPC UA) (Leitner \& Mahnke, 2006; Mahnke, Leitner, \& Damm, 2009) is such a standard in the industrial environment. OPC UA is the successor of OLE for Process Control (OPC) (Rehbein \& Pederson, 1996), which was designed to achieve cross-manufacturer machine-to-machine (M2M) communication in production environments. The address space of the OPC UA provides an ideal home for a device's virtual twin and communication based on internet protocols (IPs) offers high scalability and vertical communication.

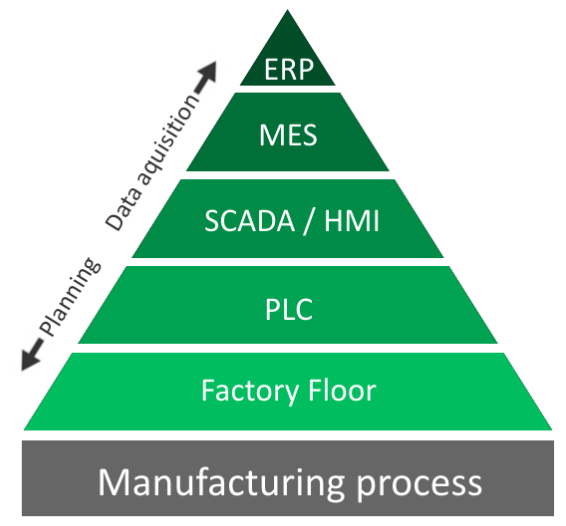

Manufacturing process

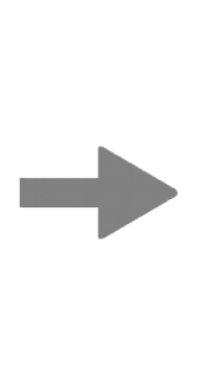

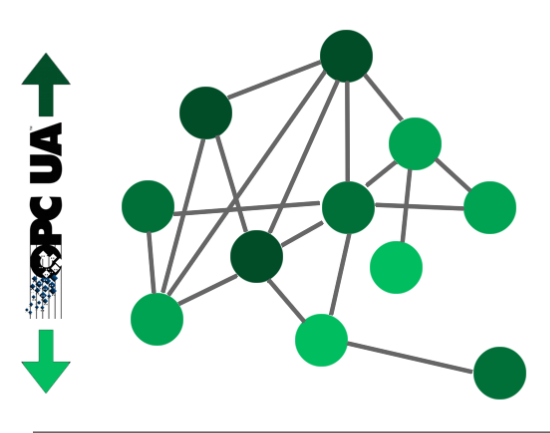

Manufacturing process

Figure 2. Dissolving the automation pyramid

Source: author.

The automation pyramid model illustrates the previously separate levels in a factory, which is now being extended with OPC UA to form an interconnected network infrastructure (Figure 2). The automation pyramid consists of hierarchical levels: communication with the 
lowest level of individual machines (factory floor) is only at the process level (programmable logic controller, PLC). In turn, the processes are controlled by the supervisory control and data acquisition (SCADA) level. Above that is the manufacturing execution system (MES) level, as part of a production management system. This level is directly linked to the operating processes and enables real-time production control. The top-level enterprise resource planning (ERP) never communicates with sensors on the factory floor. However, holistic energy management requires data from all levels of this model, both energy data from production and, for example, the use of raw materials from ERP to form EnPIs. OPC UA implements this approach of complete networking.

As OPC UA is becoming increasingly established as an important technology (Figure 3), we investigate the hypotheses of the extent to which OPC UA can also be used for CPS applications beyond typical production. We claim the framework can also drive progress in many areas other than a CPS enabler, such as building automation, smart city applications, smart grids, and energy monitoring. To support this thesis, we examine the standard in particular for its suitability to energy management and deposit the theoretical investigation with the prototypical creation of a CPS sensor.

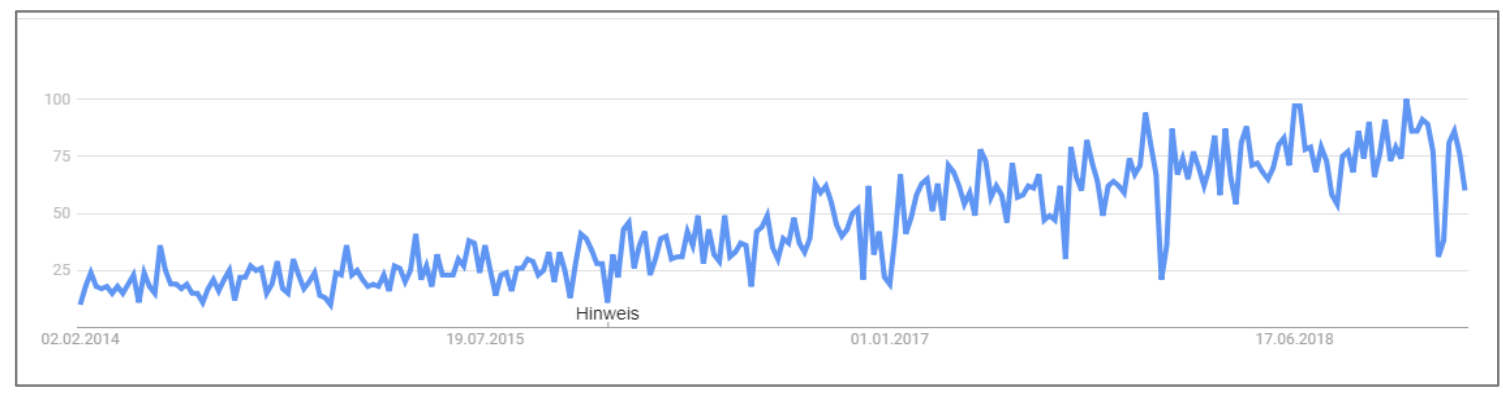

Figure 3. Five-year trend analysis of the search term 'OPC UA' (worldwide) Source: https://trends.google.com (31. Jan 2019).

\section{Methodology}

In the theoretical section, we conduct a literature review of the OPC UA technology in the context of distributed energy management and examine the standard's suitability. For this, we take a closer look at the individual parts of the OPC UA specification and discuss the possibilities and limitations of using distributed energy monitoring. In a list of requirements for a universal, networked energy management system, we align the possibilities of OPC UA with these requirements.

We demonstrate, in a quick case study, a CPS' prototypical implementation by transforming a power meter (Modbus RTU over RS485) into a CPS sensor compatible with a distributed EMS. Like a typical retrofit, we supplement the electricity meter with low-cost hardware, which gives meter with the necessary components, such as networking and virtual representation. An OPC UA server stack is our IoT platform.

\section{OPC UA for distributed energy monitoring}

\section{The OPC Unified Architecture specification}

In 2005, the OPC Foundation published the first draft of the OPC UA ("Website OPCFoundation. Available online: https://opcfoundation.org/ (accessed on 2. December 2018)," 2018). Version 1.04 contains fourteen individual documents concerning the 
standard's various aspects. The first section (OPCFoundation, 2017a) gives an overview and describes the basic concepts. The framework is standardized under IEC 62541 and offers a service-oriented architecture (SOA) that stores data models for process data (OPCFoundation, 2017b), alarms (OPCFoundation, 2017c), and historical data (OPCFoundation, 2018a) in a semantically structured address space (OPCFoundation, 2017d). The OPC UA specification defines in the address space model, as well as different node classes, basic reference types, and basic data types. The address space model is used to create the address space and is the basis of the information models. Domain-specific information models, or companion specification (CS), exist for various industries as extensions of this address space. These CS enable cross-manufacturer communication between devices from several applications, such as robotics or RFID. Other than the information models, OPC UA also describes a standardized basis for providing services (OPCFoundation, 2017e) and communication, which is possible via a client-server and a publisher/subscriber protocol (OPCFoundation, 2018b). Figure 4 shows the OPC UA stack in compact form.

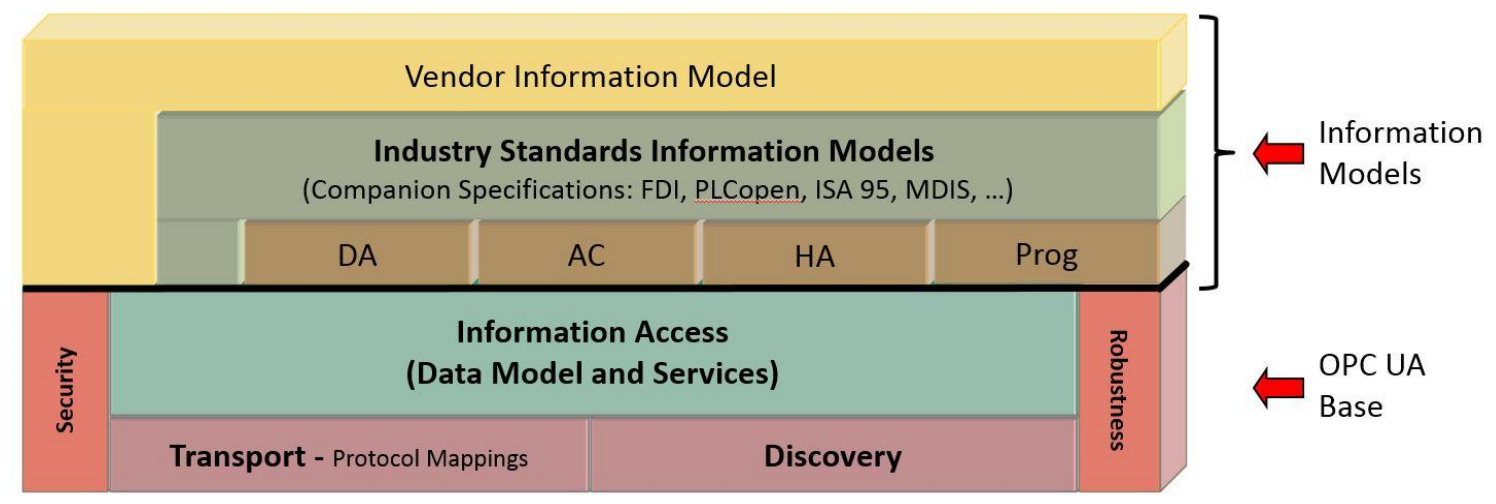

Figure 4. The OPC UA stack

Source: OPC UA Foundation (with permission)

\section{Services and service sets}

In the network, an OPC UA server at application level provides numerous services that are organized into ten service sets (OPCFoundation, 2017e). For instance, the Discovery service set allows client services to find OPC UA servers in the network. Each service set provides various services, as summarized in Table 1.

Table 1. OPC UA service sets

\begin{tabular}{|l|l|}
\hline OPC UA Service set & Description \\
\hline Discovery service set & $\begin{array}{l}\text { Services for discovering the endpoints implemented } \\
\text { by a server and determining the security } \\
\text { configuration for each of those endpoints. }\end{array}$ \\
\hline SecureChannel service set & $\begin{array}{l}\text { Services that allow a client to establish a } \\
\text { communication channel to ensure the confidentiality } \\
\text { and integrity of messages exchanged with the server. }\end{array}$ \\
\hline Session service set & $\begin{array}{l}\text { Services that allow clients to authenticate users in } \\
\text { different ways (e.g., by password or certificate) to } \\
\text { establish a session. }\end{array}$ \\
\hline
\end{tabular}




\begin{tabular}{|l|l|}
\hline NodeManagement service set & $\begin{array}{l}\text { Services that allow clients to add, delete, and modify } \\
\text { nodes in the address space. }\end{array}$ \\
\hline View service set & $\begin{array}{l}\text { Services that allow clients to browse through } \\
\text { the address space views (=subsets of the address } \\
\text { space). }\end{array}$ \\
\hline Query service set & $\begin{array}{l}\text { Services that allow clients to get a subset of data from } \\
\text { the entire address space or the view }\end{array}$ \\
\hline Attribute service set & $\begin{array}{l}\text { Services that allow clients to read and write } \\
\text { attributes (same as variables) of nodes, including } \\
\text { their historical values. }\end{array}$ \\
\hline Method service set & $\begin{array}{l}\text { Services that allow clients to call methods, including } \\
\text { specific input- and return-parameters. }\end{array}$ \\
\hline MonitoredItem service set & $\begin{array}{l}\text { Services that allow clients to create, modify, and } \\
\text { delete such called Monitoreditems used to observe } \\
\text { attributes for value changes and for events. }\end{array}$ \\
\hline Subscription service set & $\begin{array}{l}\text { Services that allow clients to create, modify, and } \\
\text { delete subscriptions on Monitoreditems }\end{array}$ \\
\hline
\end{tabular}

Source: OPC UA Specification, Part 4 (OPCFoundation, 2017e)

Some of the service sets can be used profitably for distributed energy monitoring. The Discovery service set can be used to locate sensors in the network, decoupling the role of the energy manager from the role of the technical administrator in the enterprise. The View service set is useful for integrating sensors into monitoring through an easy-to-use graphical user interface. The Attribute service set offers an important function for reading out energy data and other information, such as production quantities for EnPIs. These services are essential. The possibility of accessing historical data gives the OPC UA server the additional function of a data logger, so all data are available even if the connection is interrupted. A service for writing the data is less important for monitoring, but can be used, for example, to confirm the readings. The method service set is also irrelevant for pure energy monitoring, but for a comprehensive EMS it is needed. With this method, actuators can be switched in the system when set thresholds are exceeded, for instance, to operate load management or to switch off unnecessary consumers. The two sets for Monitoreditems and Subscriptions are interesting for energy management when a publisher/subscriber mechanism is used. This can be the case if sensor data is to be communicated to several OPC UA clients.

\section{Data types and encoding}

Another aspect of energy monitoring is possible data types. A comprehensive energy management system can process numerous units, such as meter readings (watt or kilowatt hours), fill levels (liters, kilograms), flow rates ( $\mathrm{m}^{3}$ per hour), electrical power (watts), environmental data (temperature), economic data for EnPIs (costs, quantities, people), and many more. The OPC UA address space model defines several basic data types known to every OPC UA server. (OPCFoundation, 2017f) - built-in data types are general and basic data types from computer science such as Boolean, byte, int16, int32, float, datetime or string (OPCFoundation, 2017g). However, these values are not specified by physical units. OPC UA servers can extend existing basic data types in their address space or define their own data types (Rinaldi, 2016).

OPC UA offers several options for data transport and coding. On the transport layer (OPCFoundation, 2017g), a non-standardized UA TPC protocol is defined, as well as the 
standardized protocols hypertext transfer protocol secure (HTTPS) and advanced message queuing protocol (AMQP) (OASIS-Working-Group, 2012). Data encoding is possible via a compact UA binary format (OPCFoundation, 2017g) or the data are serialized in XML or JavaScript Object Notation (Crockford, 2006). Whether TCP binary or XML-based web services are used in the application depends on the platform's performance, as embedded systems cannot always provide sufficient resources for high-performance XML processing. It should also be mentioned that OPC UA considers security protocols at the transport level.

As OPC UA does not offer any physical units or other semantic additions to numerical values in the standard, these must be defined between client and server, for example via a uniform notation of the units in the attributes. A separate CS for domain energy management would be preferable. Similar to how the OPC UA IEC 61850 Companion Specification (OPCFoundation, n.d.) maps to the IEC 61850 standard (IEC, 2013; Mackiewicz, 2006), a simple CF would have to be created for the specialist domain of energy management that considers all required parameters and units.

\section{Communication over IP}

As all transport protocols are based on the internet protocol (IP), which is ideal for networked and distributed energy monitoring, it can be used to include parts of companies and locations worldwide in monitoring and acquiring environmental data and operating data for EnPIs from higher layers of the automation pyramid or from distributed sources. With mechanisms such as virtual private networks (VPN), OPC UA services can be made available company-wide without neglecting security aspects. One disadvantage of the packet-oriented IP protocol is the lack of real-time capability; therefore, OPC UA cannot assume a process control system role. In energy monitoring, however, the timely availability of data is less critical and long-term time series are typically stored at one-minute intervals or in higher increments.

\section{Scalability}

OPC UA architecture supports combining a theoretically arbitrary number of servers and clients. In different architecture patterns, server hierarchy can be created further to the client-server relationship. Aggregating OPC UA servers can combine numerous OPC UA servers into a higher-level server by using their own client. As chained servers, they can also perform such a proxy function. Not only does this achieve resource scalability, but it also increases reliability in critical systems by redundantly maintaining multiple servers. Spatially, OPC UA scales with IP networks; thus, it is unlimited.

There is also a lot of scope: an OPC UA server can be operated as a software service in large data centers, as well as on small, embedded systems (Imtiaz \& Jasperneite, 2013). The standard also achieves this bandwidth through various server profiles that describe a different range of functions (OPCFoundation, 2017h). These four profiles build on each other step by step:

- Nano Embedded Device Server: this is the smallest possible profile including the core server, binary message encoding, and TCP support.

- Micro Embedded Device Server: this profile provides at least two simultaneous sessions and a reduced protocol for subscriptions.

- Embedded UA Server: it is possible to sign and encrypt messages in this profile. 
- Standard UA Server: this is the full-featured profile. It enables server configuration customization, enables X509 certificates, and increases the subscription volume.

From this viewpoint, the energy monitoring system as an OPC UA client and application has more potential to form the bottleneck than the OPC UA itself. A holistic energy management system must meet numerous scalability requirements for energy management. These are summarized in Table 2 and aligned with the capabilities of OPC UA.

Table 2. Scalability requirements for energy monitoring and OPC UA compliance

\begin{tabular}{|c|c|c|}
\hline Scalability dimension & Comments & Compliance by OPC UA \\
\hline Distributed resources & $\begin{array}{l}\text { The processing of potentially } \\
\text { large numbers of data sources } \\
\text { and the storage of long time } \\
\text { series of minute values should } \\
\text { be possible on distributed } \\
\text { systems. Aggregative } \\
\text { monitoring must be } \\
\text { distributable to different } \\
\text { computer systems, for example, } \\
\text { via virtualization or outsourcing } \\
\text { of short-term data logging. }\end{array}$ & $\begin{array}{l}\text { The OPC UA architecture supports the } \\
\text { distribution of UA components. Any number } \\
\text { of servers and clients can be used. Platform } \\
\text { independence allows operation in high- } \\
\text { performance environments. }\end{array}$ \\
\hline Spatial expansion & $\begin{array}{l}\text { An energy monitoring system } \\
\text { should support several } \\
\text { buildings and several locations. } \\
\text { This can reach a global } \\
\text { dimension. Hence, the system } \\
\text { must be divisible into several } \\
\text { subordinate instances. When } \\
\text { aggregating the time series of } \\
\text { energy data, for example, } \\
\text { attention must be paid to } \\
\text { different time zones. }\end{array}$ & $\begin{array}{l}\text { The framework is based on IP and the } \\
\text { architecture supports distributed } \\
\text { deployment with distributed servers and } \\
\text { clients. A discovery server helps to find } \\
\text { system-wide endpoints. }\end{array}$ \\
\hline Measuring points & $\begin{array}{l}\text { The more measuring points that } \\
\text { are integrated into the } \\
\text { monitoring, the more } \\
\text { transparent the energy flows } \\
\text { become. This number cannot } \\
\text { always be planned during } \\
\text { installation: since ISO } 50001 \\
\text { provides for continuous } \\
\text { improvement, the system must } \\
\text { support sufficient capacity for } \\
\text { several measuring points. }\end{array}$ & $\begin{array}{l}\text { There is no restriction regarding the number } \\
\text { of nodes or the size of the address space. Both } \\
\text { factors are only limited by system resources. }\end{array}$ \\
\hline Data Sources & $\begin{array}{l}\text { Integrated monitoring must be } \\
\text { able to acquire as much data as } \\
\text { possible from sensors and other } \\
\text { data sources. This requires } \\
\text { support for all standards of } \\
\text { devices (e.g., Modbus), } \\
\text { databases, and protocols. }\end{array}$ & $\begin{array}{l}\text { OPC UA plays an important role as an open } \\
\text { standard. UA can also act as a mediator to } \\
\text { non-IP-based sources, such as data from } \\
\text { fieldbus devices. With OPC UA as a data } \\
\text { source, monitoring has broad access to } \\
\text { energy data. The monitoring must have } \\
\text { implemented an OPC UA client for this } \\
\text { purpose. }\end{array}$ \\
\hline Units & $\begin{array}{l}\text { Comprehensive energy } \\
\text { monitoring should know all }\end{array}$ & $\begin{array}{l}\text { The OPC UA basic system does not know any } \\
\text { measured variables; the semantic meaning of }\end{array}$ \\
\hline
\end{tabular}




\begin{tabular}{|l|l|l|}
\hline & $\begin{array}{l}\text { common physical measurement } \\
\text { units of energy data and allow } \\
\text { defining new units. }\end{array}$ & $\begin{array}{l}\text { the values must be added by the user. } \\
\text { Companion specifications provide the } \\
\text { possibility of creating common standards. }\end{array}$ \\
\hline Interfaces & $\begin{array}{l}\text { Energy monitoring data should } \\
\text { be made available to third-party } \\
\text { systems, such as billing systems. } \\
\text { This requires standard } \\
\text { interfaces. }\end{array}$ & $\begin{array}{l}\text { The protocol is an open standard. An OPC UA } \\
\text { server, for example, can be implemented } \\
\text { within the monitoring system for the further } \\
\text { use of data from energy monitoring for third- } \\
\text { party systems. }\end{array}$ \\
\hline Users & $\begin{array}{l}\text { With a high number of users, } \\
\text { integration into existing } \\
\text { administration structures } \\
\text { (usually LDAP) should be } \\
\text { possible. }\end{array}$ & $\begin{array}{l}\text { Support user/passwords or integration in } \\
\text { any PKI, supporting trusts lists and X.501 } \\
\text { certificates. }\end{array}$ \\
\hline $\begin{array}{l}\text { Internationalization } \\
\text { and Localization }\end{array}$ & $\begin{array}{l}\text { When using the EMS across } \\
\text { national borders or with a large } \\
\text { number of users, the user } \\
\text { interface should be expandable } \\
\text { in several languages. }\end{array}$ & No relevance, as it is an M2M protocol. \\
\hline
\end{tabular}

Source: author.

\section{A quick case study: converting a power meter into a CPS}

As part of a prototypical case study, we carry out a retrofit on a classic energy meter and convert it into a CPS. As application case, we use an existing energy management system, which meets the requirements according to Table 2 and has an implemented OPC UA client, so we can build on existing work (Krutwig, Kölmel, Tantau, \& Starosta, 2019).

To realize the IP networking and as a platform for the OPC UA server, we use Raspberry Pi with a USB converter for connecting the RS485 bus. CPU of the Raspbeery Pi is a ARMv7 Processor rev 5, driven by a Debian V9.6. Figure 4 illustrates the experimental setup. The widely used Raspian (www.raspbian.org) is used as the operating system. As additional software components, a library (libmodbus.org) for reading out the RS485 and an available open-source stack for implementing the OPC UA server are used. 


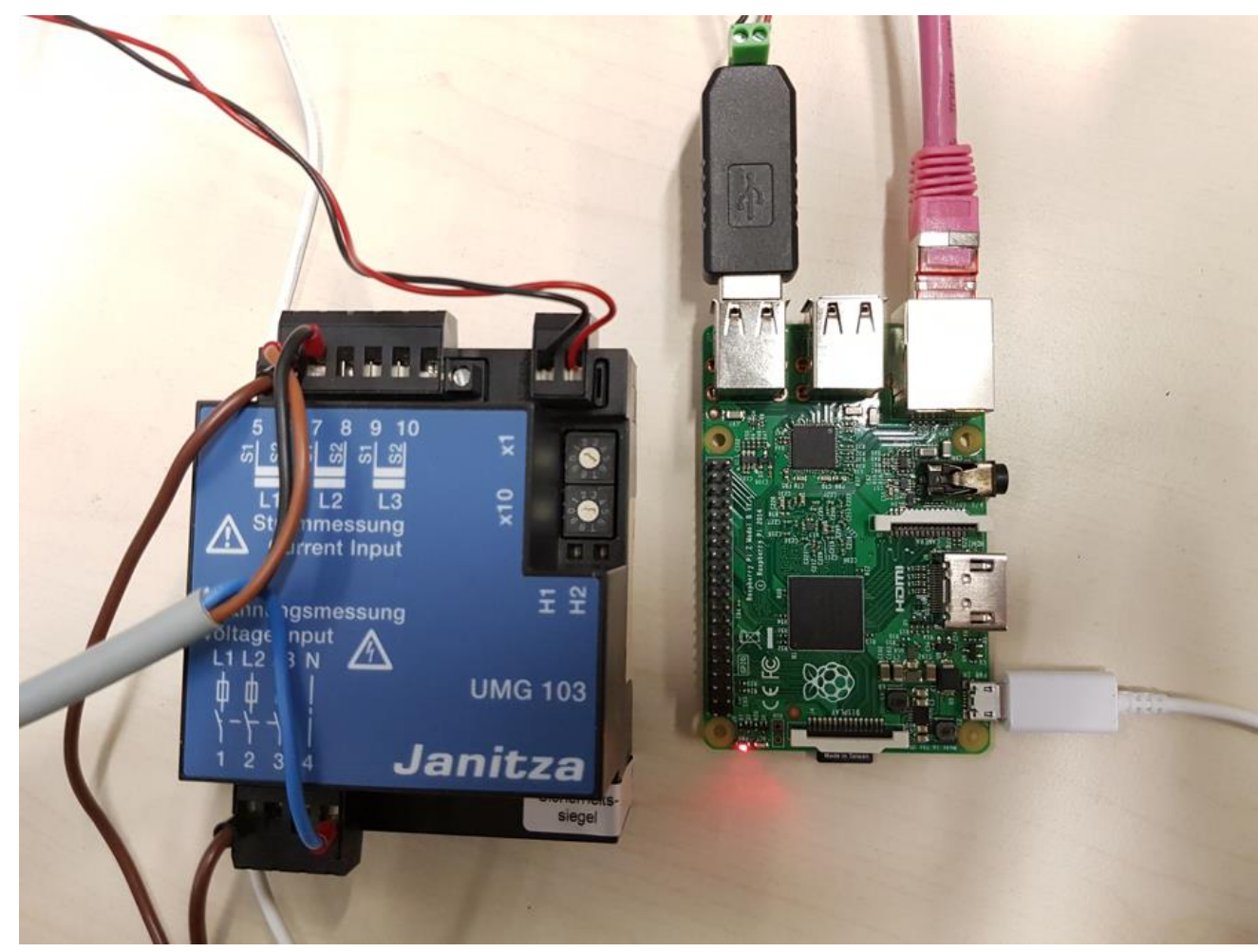

PICBE | 407

Figure 4. Prototype Setup

Source: author.

When selecting the OPC UA software, the choice is open62541 ("open62541 Website. Available online: https://open62541.org (accessed on 26. January 2019)," 2019). This is an open source C (C99) implementation of OPC UA that is licensed under the Mozilla Public License v2.0, which meets all requirements for our prototype and is already available as ready-to-use binary packages for Raspberry Pi. There is no authentication and encryption in this installed version.

The software packages were installed according to the existing documentation and required for only a few minutes. After installing the open62541 version 0.3 .0 binary packages and configuring the basic parameters, such as the network data, our OPC UA server became available in the network and could be tested via any OPC UA client. Our configuration provided a server with the Micro Embedded Device Server profile. Some operating parameters of the system were already available in the address space of the basic system. The electricity meter used is a three-phase meter whose Modbus interface is connected to a USB-RS485 converter via a two-wire connection. In order to get the data from the converter into the address space of the OPC UA server, the software library libmodbus version 3.0.6 is also used. An Ethernet connection makes the OPC UA Server available in the Intranet. With this our retrofit is complete; a CPS was made from a classic electricity meter. The meter reading can now be retrieved via any OPC UA client or via an energy management system with OPC UA client function. In contrast to RS485 networking, this process can take place worldwide as long as the IP address of the OPC UA server can be called at the respective location, for example via a Virtual Private Network (VPN). 


\section{Results}

Our literature research on OPC UA and the analysis of the standard and its properties have shown great suitability for the scope of distributed energy monitoring. In Table 2, we examined the scalability requirements of energy management systems across all dimensions and compared them with the OPC UA properties. Here, we can record the fulfillment of all scalability requirements. Weaknesses of the standard, such as a lack of support for timesensitive networks (TSN) are of little relevance for energy monitoring. Sensors that form a CPS through OPC UA are potentially available for energy monitoring, as well as for other applications for realizing intelligently networked systems (Smart Grids, Smart City, etc.). These CPS can, thus, create further added value for the company. As a result of the standard's good scalability and high flexibility, it is generally suitable to supply the cyber component in CPS formation.

We see the high application complexity as problematic when using OPC UA, because the energy manager has to find his way in the concepts of the standard, even if he only has to fetch some energy data from his viewpoint and if the switching of actuators is excluded. With some limitations, simpler IP-based IoT brokers, such as the standard message queuing telemetry transport (MQTT) (Hunkeler, Truong, \& Stanford-Clark, 2008) or the advanced message queuing protocol (AMQP) could be used instead of OPC UA. The application's complexity could be reduced if a companion specification (CS) specializing in energy management existed, with which the energy data could be supplemented by semantic information and by physical units. The OPC UA IEC 61850 companion specification (OPCFoundation, n.d.) for the energy industry under investigation points in the right direction, but cannot fulfill this task thanks to its large scope and its reduction to the energy source electricity.

Our prototypical case study gave us several insights. Meanwhile, numerous free and commercial software stacks for OPC UA are available on the market. Therefore, for every programming platform, the OPC UA service can be added quickly and easily. A sensor retrofit can then be performed with inexpensive hardware. With the universal EMS, the client's implementation is much more complex, owing to the convenient user interface. Therefore, it poses a hurdle. Nevertheless, this only has to be done once. Equipped in such a way, the EMS can then integrate all data available via the OPC UA server into the energy monitoring.

\section{Conclusion}

The OPC Unified Architecture framework is becoming increasingly widespread and accepted in industrial production. We have hypothesized that this technology is also an enabler for the Internet of Things (IoT) in other domains beyond industry and have examined this using the concrete example of distributed energy monitoring. In literature research and comparing the possibilities of OPC UA with the requirements of distributed energy management, we could show that this standard exceeds all functions and has high suitability for energy management and further fields of application. For standardized handling with OPC UA, we recommend elaborating on a dedicated companion specification (CS) for the specialist domain energy management. With our small case study, we have proven that a sensor retrofit for OPC UA can be realized quickly and cost-effectively with existing components. 


\section{References}

Baheti, R., \& Gill, H. (2011). Cyber-physical systems. The impact of control technology, 12(1), 161-166.

Crockford, D. (2006). The application/json media type for javascript object notation (json) (No. No. RFC 4627).

ElMaraghy, H. A. (2005). Flexible and reconfigurable manufacturing systems paradigms. International journal of flexible manufacturing systems, 17(4), 261-276.

Hunkeler, U., Truong, H. L., \& Stanford-Clark, A. (2008). MQTT-S-A publish/subscribe protocol for Wireless Sensor Networks. Communication systems software and middleware and workshops, 2008. comsware 2008. 3rd international conference on (pp. 791-798). IEEE.

IEC. (2013). IEC TR 61850-1:2013 Communication networks and systems for power utility automation - Part 1: Introduction and overview (Edition 2.0). IEC.

Imtiaz, J., \& Jasperneite, J. (2013). Scalability of OPC-UA down to the chip level enables Internet of Things. 2013 11th IEEE International Conference on Industrial Informatics (INDIN). IEEE. doi:10.1109/indin.2013.6622935

Jeschke, S., Brecher, C., Song, H., \& Rawat, D. B. (Eds.). (2017). Industrial Internet of Things. Springer International Publishing. doi:10.1007/978-3-319-42559-7

Krutwig, M., Kölmel, B., Tantau, A., \& Starosta, K. (2019). Standards for Cyber-Physical Energy Systems-Two Case Studies from Sensor Technology. Applied Sciences, 9(3), 435. doi:10.3390/app9030435

Leitner, S.-H., \& Mahnke, W. (2006). OPC UA-service-oriented architecture for industrial applications. ABB Corporate Research Center.

Mackiewicz, R. E. (2006). Overview of IEC 61850 and Benefits. 2005/2006 PES TD. IEEE. doi:10.1109/tdc.2006.1668522

Mahnke, W., Leitner, S.-H., \& Damm, M. (2009). OPC Unified Architecture. Springer Berlin Heidelberg. doi:10.1007/978-3-540-68899-0

Monostori, L., Kádár, B., Bauernhansl, T., Kondoh, S., Kumara, S., Reinhart, G., Sauer, O., et al. (2016). Cyber-physical systems in manufacturing. CIRP Annals, 65(2), 621-641. doi:10.1016/j.cirp.2016.06.005

OASIS-Working-Group. (2012). Advanced Message Queuing Protocol (AMQP) Version 1.0. OASIS Open.

OPCFoundation. (2017a). OPC UA Specification: Part 1 - Concepts, Core Specification Parts, Version 1.04. OPC Foundation. Retrieved from https://opcfoundation.org

OPCFoundation. (2017b). OPC UA Specification: Part 8 - Data Access, Access Type Specification Parts, Version 1.04. OPC Foundation. Retrieved from https://opcfoundation.org

OPCFoundation. (2017c). OPC UA Specification: Part 9 - Alarms \& Conditions, Access Type Specification Parts, Version 1.04. OPC Foundation. Retrieved from https://opcfoundation.org

OPCFoundation. (2017d). OPC UA Specification: Part 3 - Address Space Model, Core Specification Parts, Version 1.04. OPC Foundation. Retrieved from https://opcfoundation.org

OPCFoundation. (2017e). OPC UA Specification: Part 4 - Services, Core Specification Parts, Version 1.04. OPC Foundation. Retrieved from https://opcfoundation.org 
OPCFoundation. (2017f). OPC UA Specification: Part 5 - Information Model, Core Specification Parts,Version 1.04. OPC Foundation. Retrieved from https://opcfoundation.org OPCFoundation. (2017g). OPC UA Specification: Part 6 - Service Mappings, Core Specification Parts,Version 1.04. OPC Foundation. Retrieved from https://opcfoundation.org OPCFoundation. (2017h). OPC UA Specification: Part 7 - Profiles, Core Specification Parts, Version 1.04. OPC Foundation. Retrieved from https://opcfoundation.org OPCFoundation. (2018a). OPC UA Specification: Part 11 - Historical Access, Access Type Specification Parts, Version 1.04. OPC Foundation. Retrieved from https://opcfoundation.org

OPCFoundation. (2018b). OPC UA Specification: Part 14 - Pub Sub, Version 1.04. OPC Foundation. Retrieved from https://opcfoundation.org

OPCFoundation. (n.d.). OPC UA IEC 61850 Companion Specification, Release Candidate Version 1.0, February 5th, 2018. OPC Foundation. Retrieved from https://opcfoundation.org

open62541 Website. Available online: https://open62541.org (accessed on 26. January 2019). (2019). Retrieved from https://open62541.org/

Rao, P., Muller, M. R., \& Gunn, G. (2017). Conducting a metering assessment to identify submetering needs at a manufacturing facility. CIRP Journal of Manufacturing Science and Technology, 18, 107-114. doi:10.1016/j.cirpj.2016.10.005

Rehbein, D. A., \& Pederson, A. (1996). Impact of the OLE for Process Control (OPC) standard on the process industry. Proceedings of the Industrial Computing Conference (Vol. 6, pp. 285-293).

Rinaldi, J. S. (2016). OPC UA - Unified Architecture: The Everyman's Guide to the Most Important Information Technology in Industrial Automation. CreateSpace Independent Publishing Platform.

Schlick, J. (2012). Cyber-physical systems in factory automation-Towards the 4th industrial revolution. Factory Communication Systems (WFCS), 2012 9th IEEE International Workshop on (pp. 55-55). IEEE.

Standardization, I. O. for. (2018). ISO 50001:2018 Energy management systems Requirements with guidance for use (Vol. 2008). Geneva, CH: International Organization for Standardization.

Website OPCFoundation. Available online: https://opcfoundation.org/ (accessed on 2. December 2018). (2018). Retrieved from https://opcfoundation.org/

Wolf, W. H. (2009). Cyber-physical systems. IEEE Computer, 42(3), 88-89. doi:10.1109/MC.2009.81 